\title{
Watermark Resynchronization: An Efficient Approach Based on Eulerian Tours around a Robust Skeleton
}

\author{
Konstantinos Raftopoulos ${ }^{1}$, Klimis Ntalianis ${ }^{2}$, Paraskevi Tzouveli ${ }^{3}$, \\ Nicolas Tsapatsoulis ${ }^{4}$, Aleatha Parker-Wood ${ }^{1,5}$, and Marin Ferecatu ${ }^{1}$ \\ ${ }^{1}$ École SITI, Conservatoire National des Arts et Métiers, \\ 292 Rue St Martin FR-75141 Paris Cedex 03, France \\ \{konstantinos.raftopoulos, marin. ferecatu\}@cnam. fr \\ ${ }^{2}$ Technological Educational Institute of Athens, Department of Marketing - \\ Online Computing Group, Egaleo 12242, Athens, Greece \\ kdal75@gmail.com \\ ${ }^{3}$ National Technical University of Athens, Electrical and Computer Engineering Department, \\ Zografou 15773, Athens, Greece \\ tpar@image.ntua.gr \\ ${ }^{4}$ Cyprus University of Technology, Department of Communication and Internet Studies \\ Limassol, CYPRUS \\ nicolas.tsapatsoulis@cut.ac.cy \\ ${ }^{5}$ University of California Santa Cruz, Storage Systems Research Center, \\ Santa Cruz, California USA \\ aleatha@soe.ucsc.edu
}

\begin{abstract}
The existing block-based approaches, albeit successful in resisting frequency domain attacks, are sensitive to geometric distortions due to the lack of reference in repositioning the block grid. In this paper an RST-invariant block-based approach is proposed, aiming at protecting image objects. The term "image object" refers to semantically contiguous parts of images that have a specific contour boundary. The proposed approach is based on shape information, since the watermark is embedded in image blocks, the location and orientation of which are defined by Eulerian tours that are appropriately arranged in layers, around an object's robust skeleton. Images from the Polymathic project are used to illustrate the technique.
\end{abstract}

Keywords: Object-based Watermarking, Resynchronization, Skeleton Transform, Global-Local Transformation, Eulerian Tours.

\section{Introduction}

Block-based watermarking approaches using spread spectrum modulation of pseudorandom signals, even though successfully improving watermark resistance to various frequency domain attacks, cannot withstand rotation, because the rectangular grid arrangement is changed and thus synchronization is lost. A reference point for placing the rectangular block-grid for watermark recovery is not easy to obtain after image/object rotation, if a priori registration information is not available. The situation 
is illustrated in [1] where elaborate methods of high complexity are proposed to alleviate this problem. The main contribution of this paper is that it incorporates a certain noise resistant method of extracting shape information[2] during the block-based watermark embedding process in such a way that the watermarked block's location is readily identifiable at the retrieval phase, even after RST transformation, cropping or Gaussian boundary noise. The proposed system is tested in its ability to provide geometrically resistant copyright protection of semantic objects having an explicit boundary, most suited for protecting certain objects in an image, or explicit creations of artists that have to be distributed and reused in different contexts. Another application is inferring image provenance, allowing artists to find source images quickly by examining watermarks in derivative files, similar to activity inference [3].

\section{The Method}

A robust skeleton of each object is initially extracted in a vector form. To introduce enhanced noise resistance to the extracted skeleton, we use the Global-Local transformation [2] to map the noise contour back to a smoothed version. Starting then from the skeleton endpoints (marked as small circles in the images below), the pseudorandom watermark sequence is embedded in the DCT domain of the blocks along the skeleton's Eulerian tour. When the first tour is completed the Eulerian tour is extended outwards, in consecutive layers towards the object's boundary until the watermark sequence is spread to the whole object. During watermark detection, initially the skeleton of the candidate object is extracted and the potentially watermarked blocks that are located along the extended Eulerian tour are matched against the respective blocks around the initial skeleton. Only tours starting from the skeleton endpoints are examined. The success of the method stems from the fact that based on the Global-Local transformation, the robust skeleton is extracted from a smooth version of the original contour that retains certain metric information.

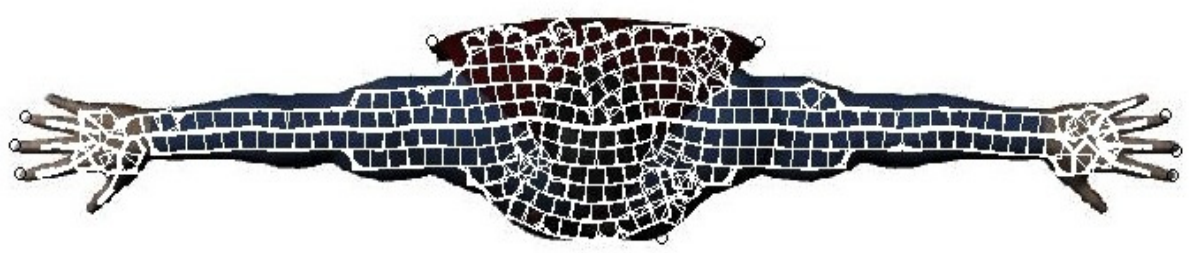

Fig. 1. Watermarked blocks arranged across the Eulerian tours around the skeleton of the Superman top view 


\section{Experiments}

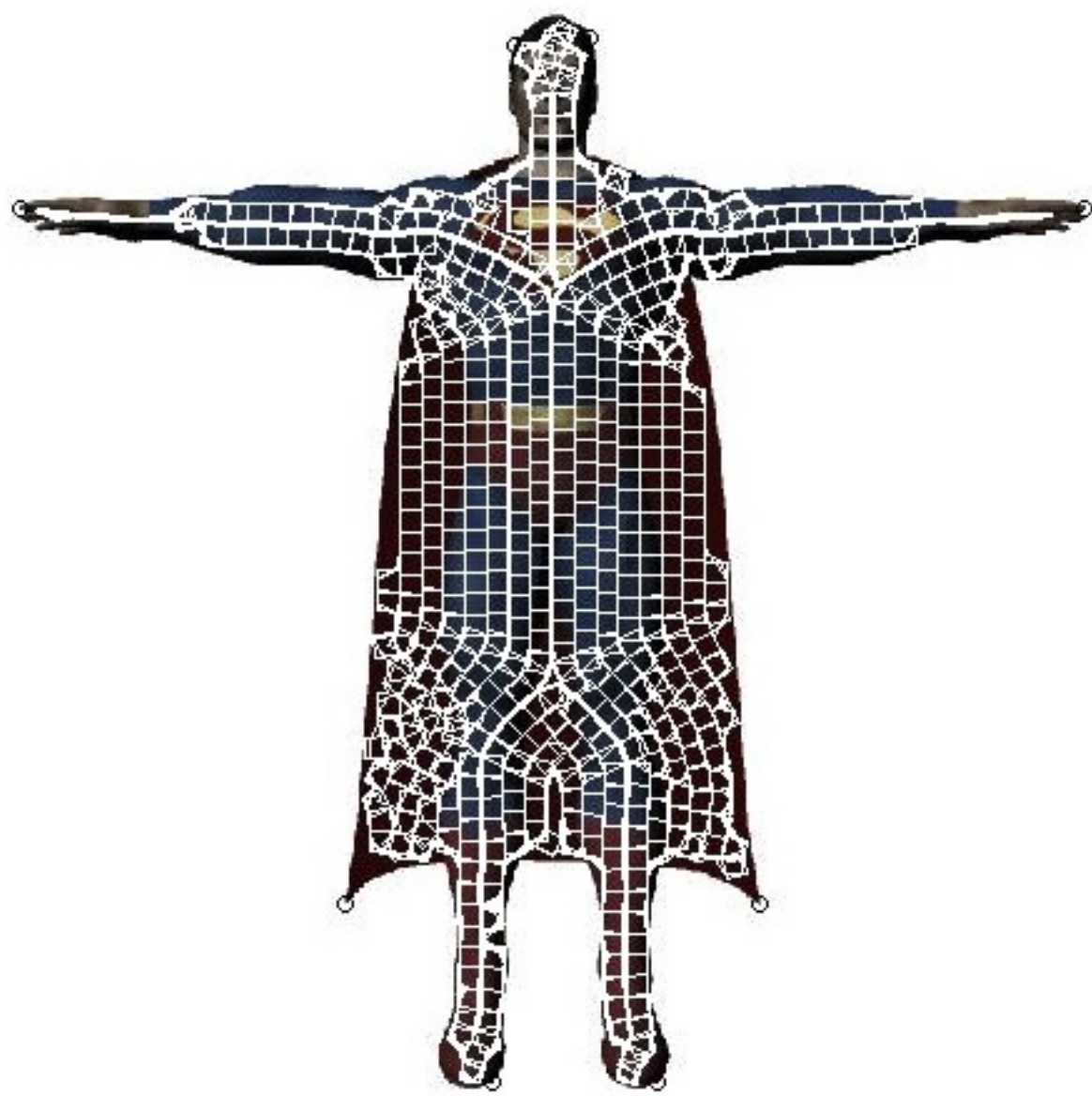

Fig. 2. The location of the watermarked blocks arranged in layers around the skeleton is illustrated for the Superman figure. 


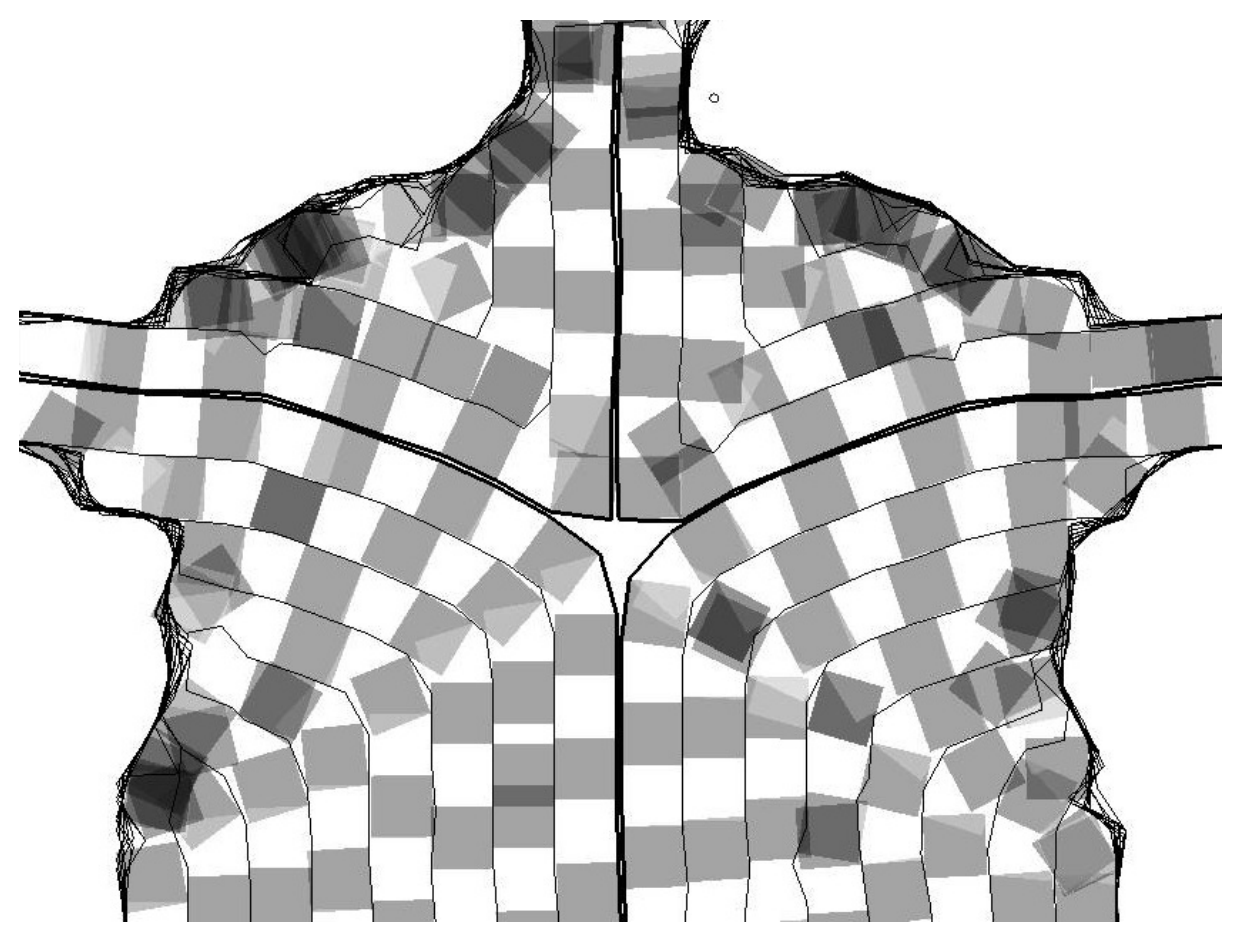

Fig. 3. A closer view of the Eulerian tours at the back view of the Superman image. Some overlap has been allowed for better illustration

Acknowledgements. This research has been supported by the Project FUI Polymathic, financed by the French government.

\section{References}

1. Lin, C., Wu, M., Bloom, J., Cox, I., Miller, M., Lui, Y.: Rotation, scale, and translation resilient watermarking for images. IEEE Transaction on Image Processing 10(5), 767-782 (2001)

2. Raftopoulos, K.A., Kollias, S.D.: The Global-Local transformation for noise resistant shape representation. Computer Vision and Image Understanding 115(8), 1170-1186 (2011)

3. Gaugaz, J., Costache, S., Chirita, P.A., Firan, C.S., Nejdl, W.: Activity based links as a ranking factor in semantic desktop search. In: Latin American Web Conference, IEEE LAWEB 2008, pp. 49-57 (2008) 